\title{
Temporal Dynamics in COVID-19 Transmission: Case of Some African Countries
}

\author{
Alemtsehai A. Turasie \\ New Jersey City University, Jersey City, USA \\ Email:alemtsehaia@yahoo.co.uk
}

How to cite this paper: Turasie, A.A. (2020) Temporal Dynamics in COVID-19 Transmission: Case of Some African Countries. Advances in Infectious Diseases, 10, 110-122.

https://doi.org/10.4236/aid.2020.103011

Received: July 1, 2020

Accepted: August 3, 2020

Published: August 6, 2020

Copyright (อ 2020 by author(s) and Scientific Research Publishing Inc. This work is licensed under the Creative Commons Attribution International License (CC BY 4.0).

http://creativecommons.org/licenses/by/4.0/ (c) (i) Open Access

\begin{abstract}
Coronavirus disease 2019 (COVID-19) has become a global threat to public health and economy. The potential burden of this pandemic in developing world, particularly the African countries, is much concerning. With the aim of providing supporting evidence for decision making, this paper studies the dynamics of COVID-19 transmission through time in selected African countries. Time-dependent reproduction number $\left(R_{t}\right)$ is one of the tools employed to quantify temporal dynamics of the disease. Pattern of the estimated reproduction numbers showed that transmissibility of the disease has been fluctuating through time in most of the countries included in this study. In few countries such as South Africa and Democratic Republic of Congo (DRC), these estimates dropped quickly and stayed stable, but greater than 1, for months. Regardless of their variability through time, the estimated reproduction numbers remain greater than or nearly equal to 1 in all countries. Another Statistical model used in this study, namely Autoregressive Conditional Poisson (ACP) model, showed that expected (mean) number of new cases is significantly dependent on short range change in new cases in all countries. In countries where there is no persistent trend in new cases, current mean number of new cases (on day $t$ ) depend on both previous observation and previous mean (day $t-1)$. In countries where there is continued trend in new cases, current mean is more affected by number of new cases on preceding day.
\end{abstract}

\section{Keywords}

COVID-19, Reproduction Number, Autoregressive Conditional Poisson, Daily New Cases, Time Series, SARS-CoV-2, COVID-19 in Africa

\section{Introduction}

The World Health Organization (WHO), on March 11 2020, declared the out- 
break of novel coronavirus (COVID-19) a pandemic [1]. Since then the virus that causes coronavirus, SARS-CoV-2, has spread to every continent causing incalculable public health and economic disaster. From mitigation and prevention point of view, the burden of this pandemic is even heavier on developing world, particularly the African countries. Therefore, studying the dynamics of this pandemic in these countries is of great importance in terms of providing indicators that support informed decision making.

Studies report that Africa has made substantial progress since Ebola outbreak and prepared better for COVID-19 [2]. These studies claim that some African nations implemented promising measures well before any cases of COVID-19 had been reported from Africa. For instance, countries such as Nigeria, Kenya, Ethiopia, Côte d'Ivoire, Ghana, Uganda and Botswana have been acknowledged, in the cited source, for rapidly dealing with suspected cases. That being an encouraging development, this study examines temporal and partly spatial dynamics of COVID-19 since reported in some African countries. Visualizing the infectiousness pattern of the disease across time and locations is vital noting that variation may occur depending on contact rate of the source cases in communities with differing population density and culture. As many agree, better understanding of the spread and transmission dynamics of infectious disease like COVID-19 is key in determination of effective response measures.

To have continent-wide overview, countries from the five regions of the continent: South Africa from Southern Africa region; Nigeria, Côte d'Ivoire, and Guinea from Western Africa; Democratic Republic of Congo (DRC) and Gabon from Central Africa; Ethiopia, Kenya, Sudan and Djibouti from Eastern Africa; Algeria and Egypt from Northern Africa are included in this study. Inclusion of Djibouti has a specific reason. From visual assessment of epidemic curve, this country looks to be facing a second wave of the pandemic. The spread of COVID19 in Djibouti appeared to be contained around the end of April (for relatively longer time) and again rose up in mid May. Behavior of estimates within and across such cycles can be of interest and may serve as a benchmark in interpreting patterns of similar estimates in other countries.

\section{Data}

Daily time series of total new cases from WHO's daily situation reports [3] are used in this study. The author chose this source for data quality and reliability. The data were extracted manually from separate daily reports. The daily counts are as reported by the respective national authorities to the WHO by $10 \mathrm{AM}$, Geneva time, each day. Length of the time series differs for different countries, see Table 1 . This is so because first case(s) of COVID-19 were reported on different times/dates in different countries.

\section{Reproduction Number}

Reproduction number is one of the measures of transmissibility of infectious 
Table 1. Length of time series used for each country-time between date on which the first case is recorded (by the WHO) and June 15, 2020.

\begin{tabular}{ll}
\hline Country & \multicolumn{1}{c}{ Time period covered } \\
\hline South Africa & $2020-03-06$ to $2020-06-15$ \\
DRC & $2020-03-11$ to $2020-06-15$ \\
Gabon & $2020-03-14$ to $2020-06-15$ \\
Nigeria & $2020-02-28$ to $2020-06-15$ \\
Côte d'Ivoire & $2020-03-12$ to $2020-06-15$ \\
Guinea & $2020-03-14$ to $2020-06-15$ \\
Ethiopia & $2020-03-14$ to $2020-06-15$ \\
Kenya & $2020-03-14$ to $2020-06-15$ \\
Sudan & $2020-03-14$ to $2020-06-15$ \\
Djibouti & $2020-03-19$ to $2020-06-15$ \\
Algeria & $2020-02-26$ to $2020-06-15$ \\
Egypt & $2020-02-15$ to $2020-06-15$ \\
\hline
\end{tabular}

disease. It is often used to reflect how infectious a disease is. In general, it refers to the average number of secondary cases caused by an infected individual. In practice, different qualifiers are used when reporting reproduction number: initial, basic, instantaneous... etc [4] and several estimation techniques are proposed in literature (e.g., [5] [6] [7] [8]).

There are intervention measures (such as social distancing, isolation, personal hygiene) put in place in different countries. Moreover, some proportion of the population can be immune to COVID-19 (for example due to prior infection). Therefore, time-dependent reproduction number is more appropriate to visualize dynamics of the pandemic with time. In this paper, I analyze time-dependent (instantaneous) reproduction number $\left(R_{t}\right)$ using method discussed in [8]. This method assumes that, once infected, individuals have an infectivity profile given by a probability distribution $w_{s}$, which is dependent on time, $s$, since infection of the case. It is believed that the distribution $w_{s}$ typically depends on individual biological factors such as pathogen shedding or symptom severity. Studies have shown that $W_{s}$ for SARS-CoV-2, the virus that causes COVID-19, peaks just before or as patients developed symptoms [9] [10].

Let $I_{t}$ be total number of incident cases arising at time $t$ and let $\mathcal{T}_{t}$ be total infectiousness of infected individuals at time $t, \mathcal{T}_{t}=\sum_{s=1}^{t} I_{t-s} w_{s}$ (the sum of infection incidence up to time step $t-1$, weighted by the infectivity function $w_{s}$ ). Given these, [8] proposed that $R_{t}$ can be estimated by the ratio of $I_{t}$ to $\mathcal{T}_{t}$.

$R$ packages, "EpiEstim" and "incidence" are employed to compute estimates of $R_{t}$ in this study. These calculations are based on daily incidence time series and two parameters (namely, mean and standard deviation) of serial interval distribution. Different estimates of mean and standard deviation of serial interval for COVID-19 are reported by different authors (e.g., [9] [11] [12] [13] [14] 
[15]). The estimates provided by [11] $($ mean $=7.0$ days and standard deviation $=$ 4.5 days) use in this study.

Epidemic curves and the estimated time-varying reproduction numbers, for the countries considered in this study, are given in Figure 1 and Figure 2 respectively. Studying these two results jointly may also help to evaluate capability of $R_{t}$ to capture the observed dynamics. From Figure 2, we can see that reproduction number of the pandemic has been lowered quickly and kept somehow stable in countries such as South Africa and DRC. However the estimates remain notably above 1 in these countries as well, showing that still more cases are expected with one agent infecting on average more than 1 individuals.

On the other hand, the estimates of $R_{t}$ values fluctuate with time in the rest of countries with temporary ups and downs. Countries like Nigeria, Guinea, Sudan and Egypt had a relatively lower early transmissibility rate which quickly rose up before dropping back after a couple of weeks. In contrast, countries such as Ethiopia, Djibouti and Algeria had higher initial transmissibility rate which quickly, but temporarily, dropped within a week or two. These patterns may indicate how relatively reluctant or aggressive response measures were taken by the countries at initial stage of the pandemic. Regardless of stability or fluctuation with time, the estimated values $R_{t}$ remain above or very close to 1 in almost all countries. This may indicate that there is a need for continued and/or improved preventive intervention in these countries. This recommendation makes sense because the temporal dynamics of estimated values of $R_{t}$ can have implications on effectiveness of response measures in place [16] [17]. Magnitude of these estimates also play an important role in the selection and aggressiveness of countermeasures (e.g. social distancing, travel restrictions, handling infected agents and so on) required to contain the spread COVID-19 [18]. Note that the estimated values of $R_{t}$ are momentarily lower than 1 in countries such as Gabon, Guinea and Djibouti at the end of the study period, June 15. However, quality of data from these countries, particularly Gabon, seems to be questionable and this may affect reliability of the estimates. As can be seen from epidemic curve in Figure 1, relatively very high number of new cases are followed by 0 new cases, specifically in Gabon. Estimates based on data with such inconsistency may not reflect the actual contagion of the virus. Therefore, the author suggests that results for countries with inconsistent incidence pattern should be interpreted with light limitations.

The epidemic curve and reproduction number for Djibouti show that the situation seemed to be contained in April 20's. During this time reproduction number has been less than 1 for several days in a row. However, it started rising up again since mid May and reached a peak of 4.14 on May 21. This indicates that the spread of the virus in Djibouti looks to be in its second cycle.

Projected number of new cases for the next 30 days, June 16-July 15, are given in Figure 3. These projections are made using the median level of estimated $R_{t}$ values, shown in Table 2 , for each country and assuming that $R_{t}$ value may not 
deviate significantly over the next 30 days. An $R$ package called "projections" is used to compute these projections. Plausibility of the projections is briefly assessed dividing the data into training and test data. The author would also like
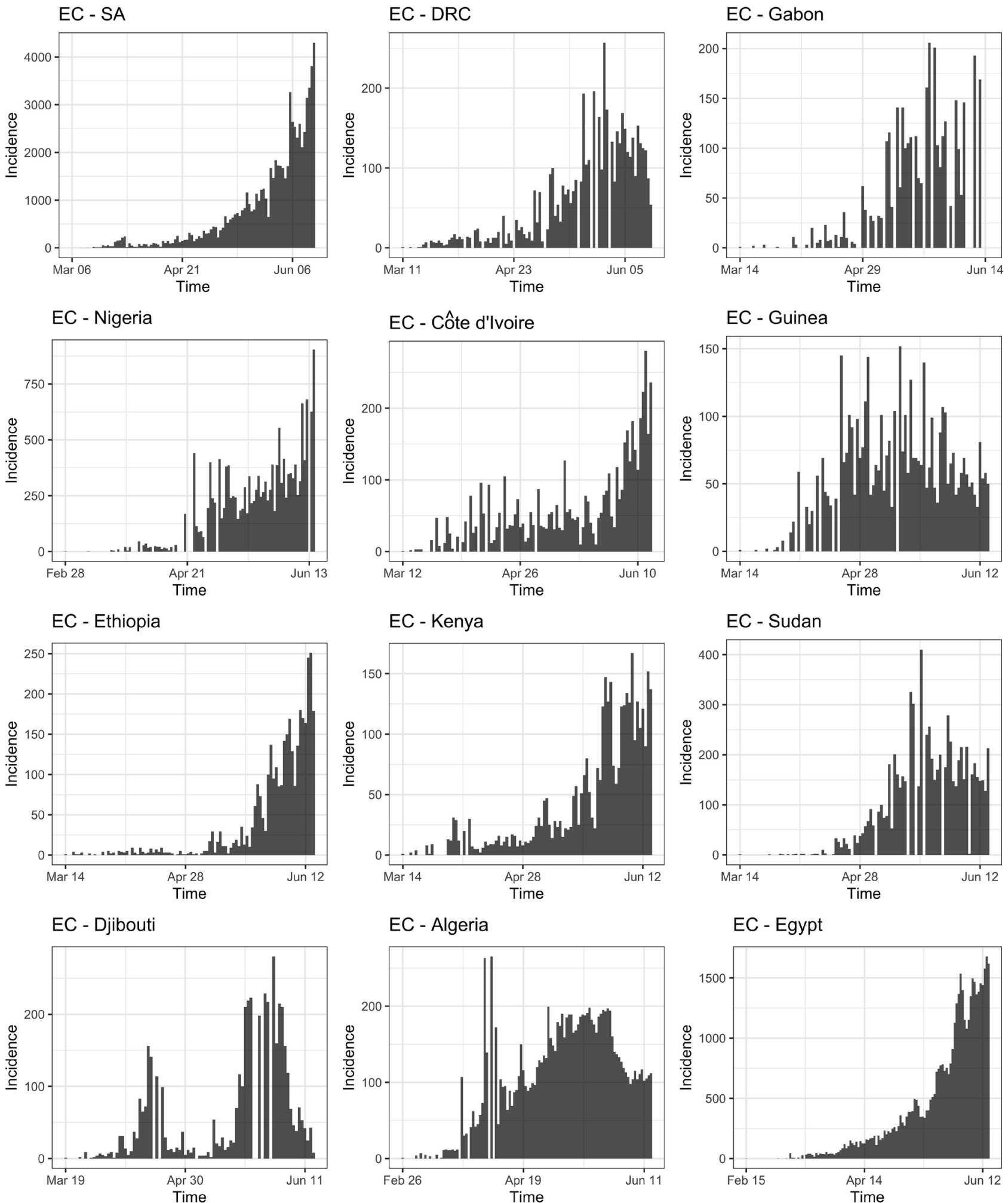

Figure 1. Epidemic curves of COVID-19 for the countries included in this study. Note: EC stands for Epidemic Curve. 

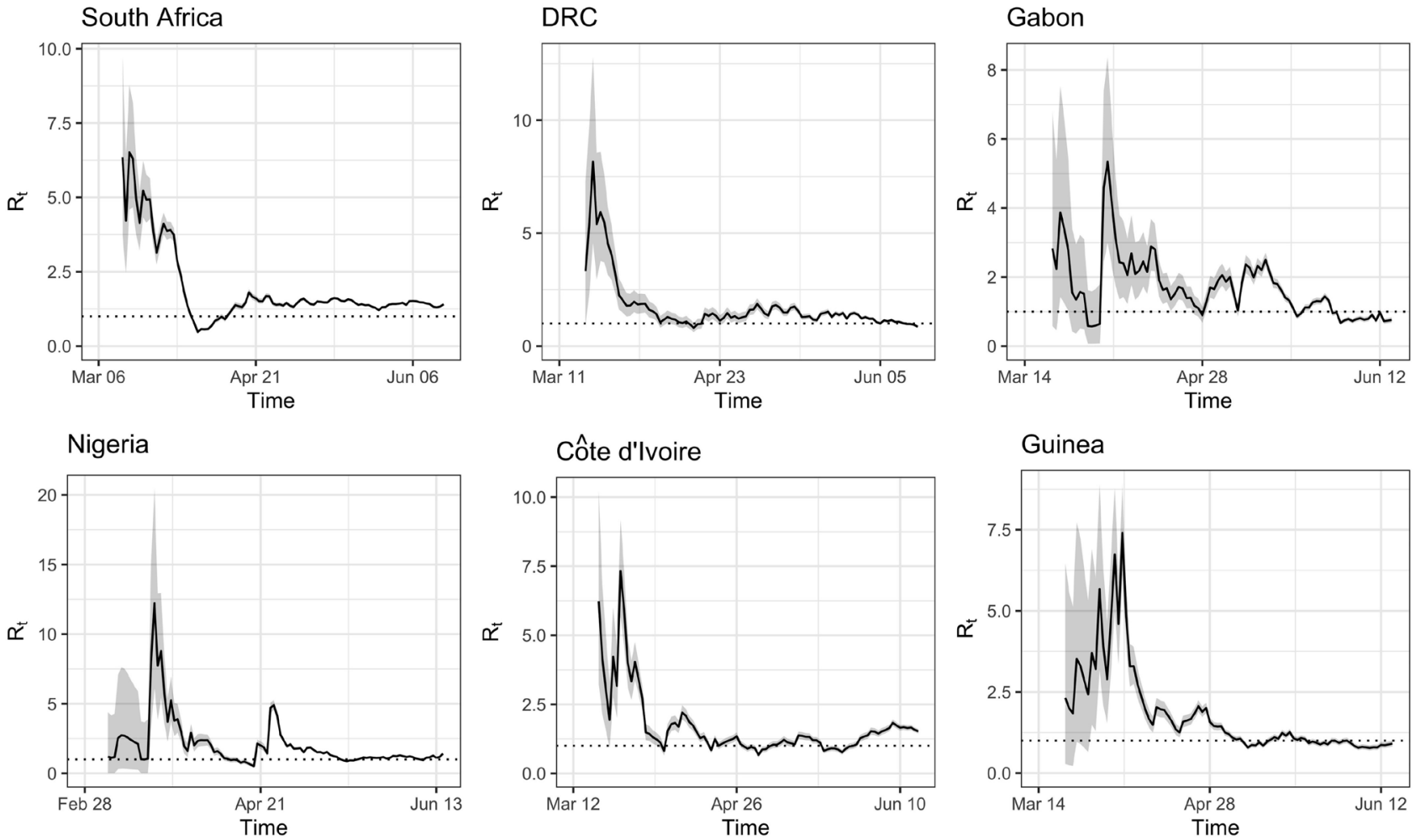

Côte d'Ivoire
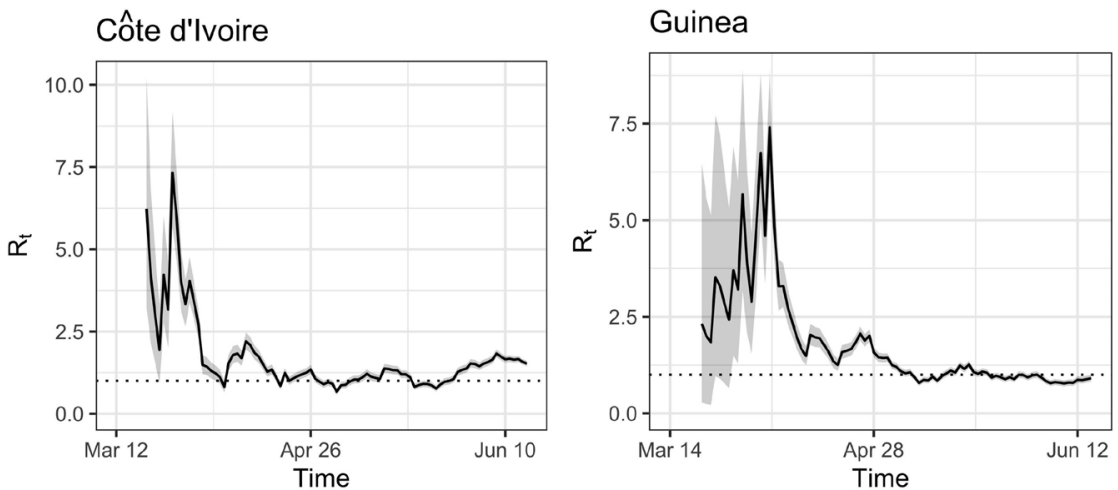

Ethiopia

\section{Kenya}
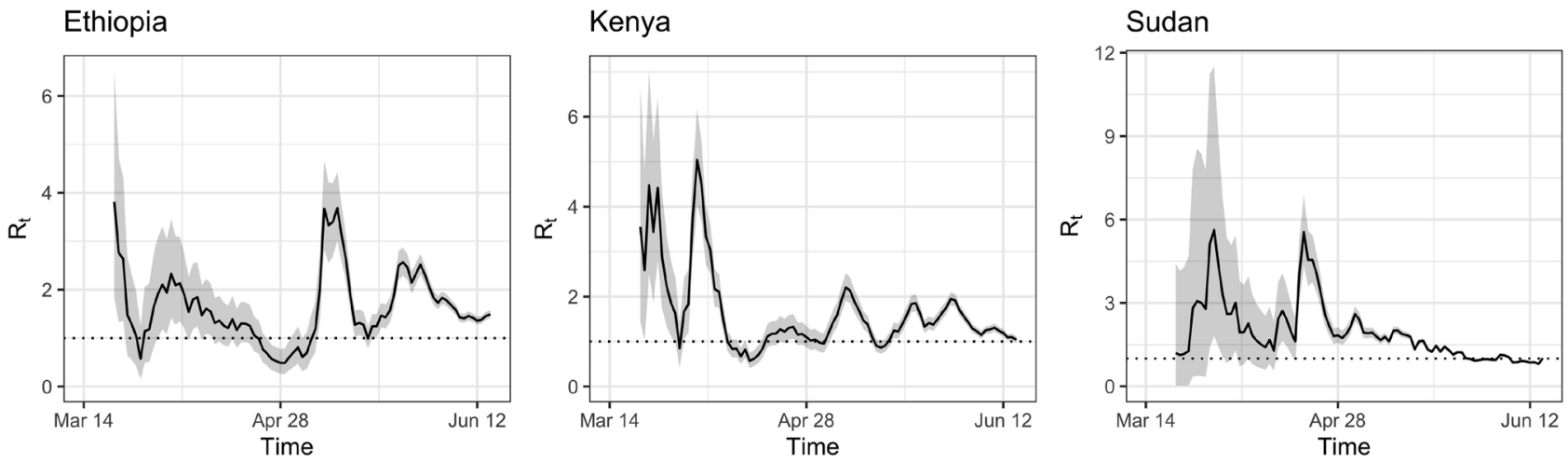

Djibouti

\section{Algeria}
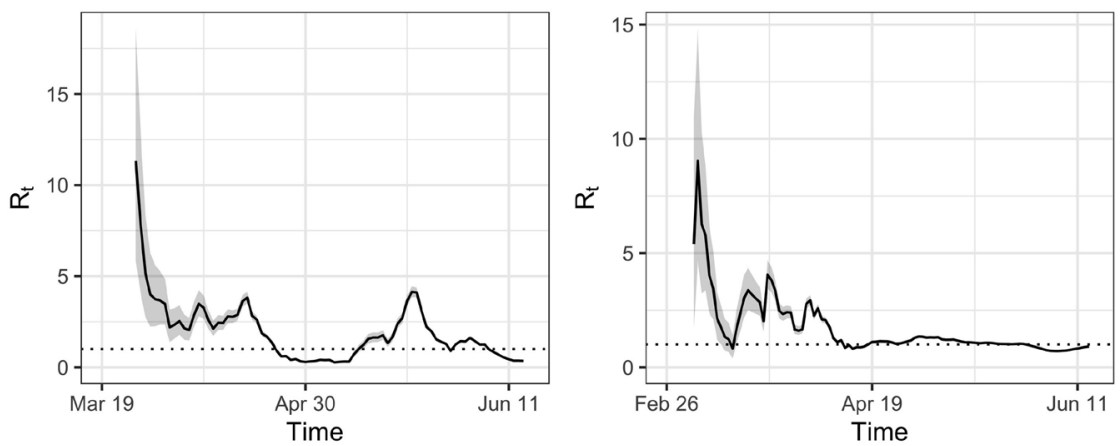

\section{Egypt}

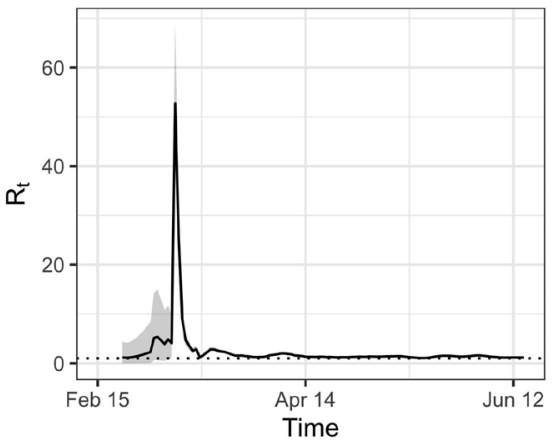

Figure 2. Estimates of time-dependent reproduction number of COVID-19 over weekly sliding window ending on June 15, 2020. Gray shades are the $95 \%$ confidence limits. Note that these credible intervals get wider when case numbers get low. 
South Africa

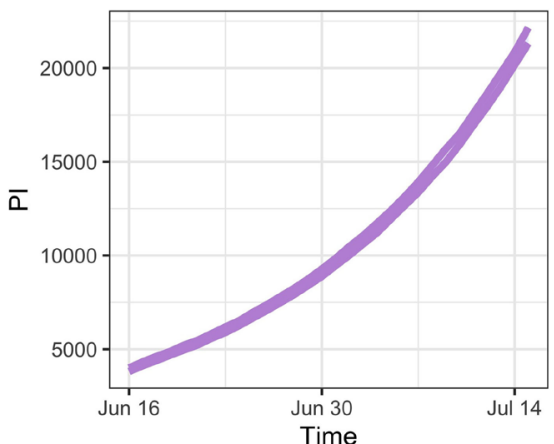

Nigeria

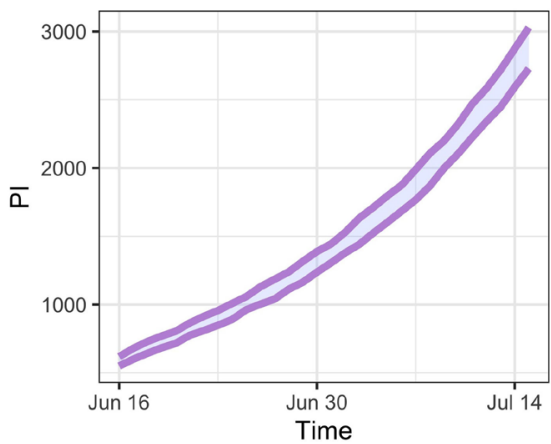

Ethiopia

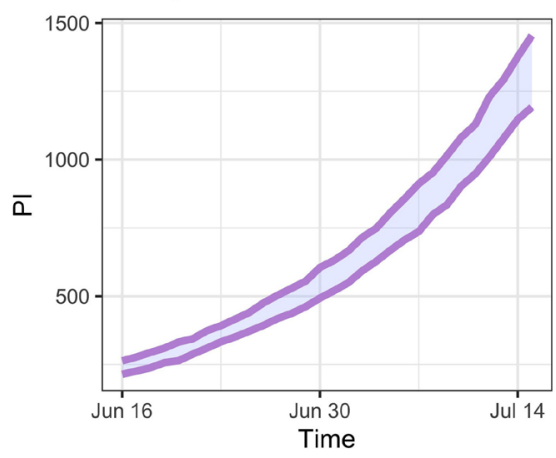

Djibouti

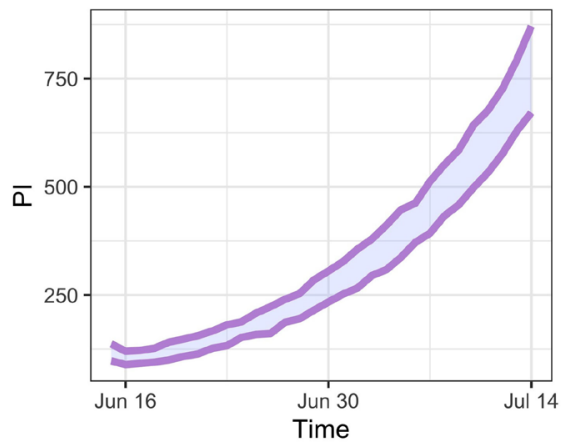

DRC

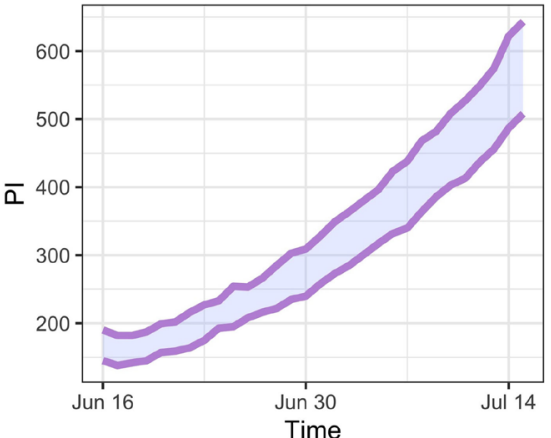

Côte d'Ivoire

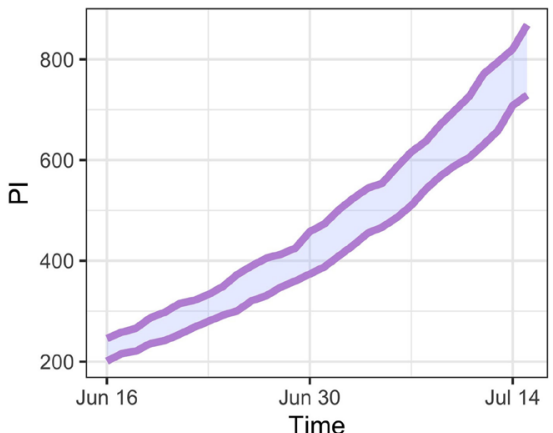

Kenya

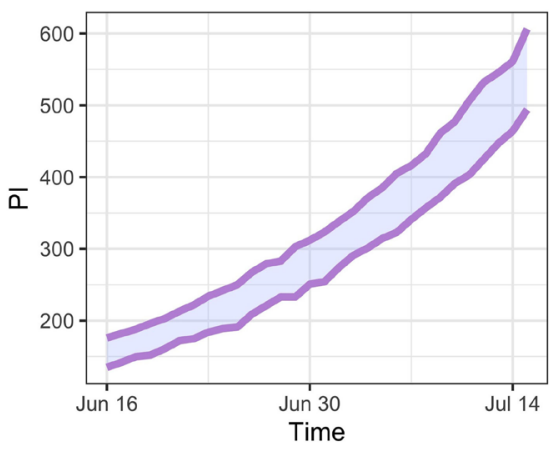

Algeria

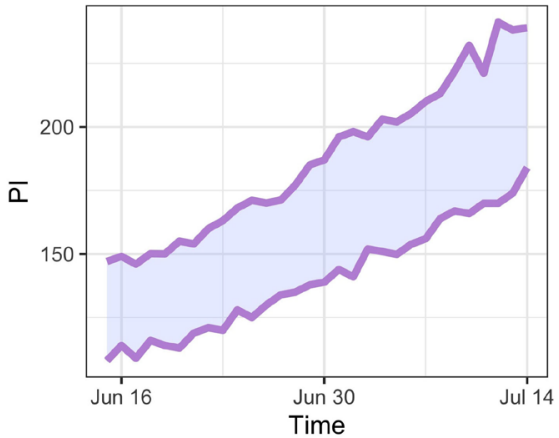

Gabon

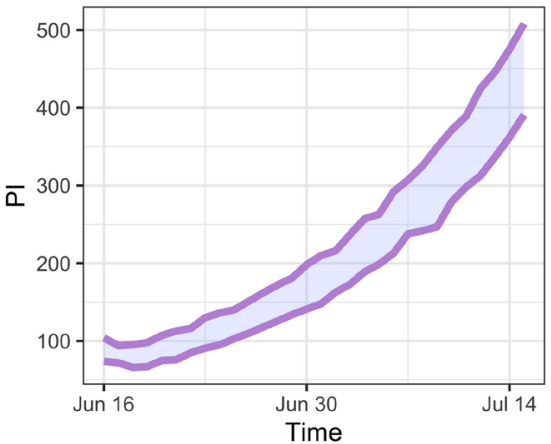

Guinea

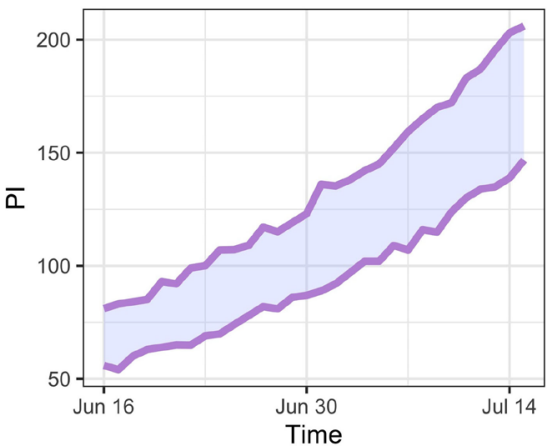

Sudan

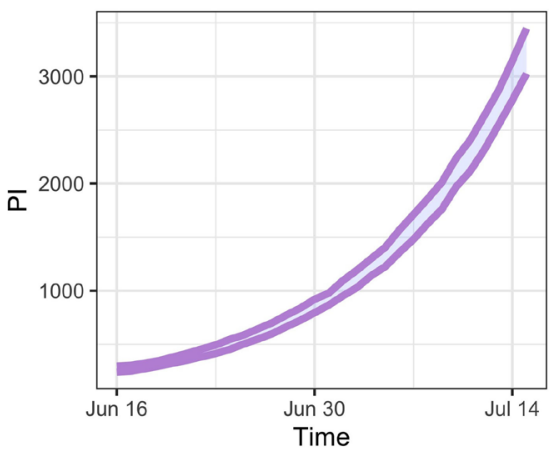

Egypt

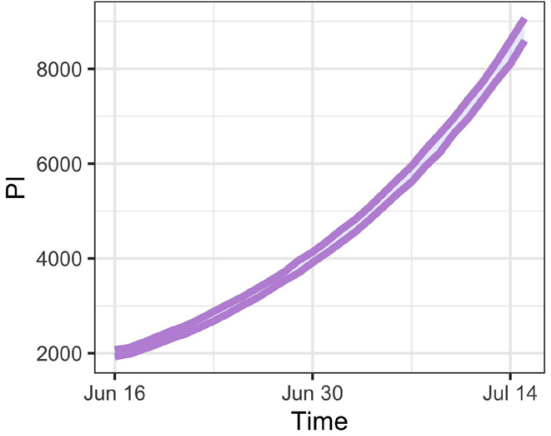

Figure 3. Projected incidences, 95\% confidence intervals, for the next 30 days, June 16-July 15 . Note that PI stands for Predicted Incidences. 
Table 2. Median level of estimated time-dependent reproduction numbers.

\begin{tabular}{lc}
\hline Country & Median reproduction number \\
\hline South Africa & 1.46 \\
DRC & 1.37 \\
Gabon & 1.51 \\
Nigeria & 1.41 \\
Côte d'Ivoire & 1.33 \\
Guinea & 1.25 \\
Ethiopia & 1.47 \\
Kenya & 1.33 \\
Sudan & 1.74 \\
Djibouti & 1.61 \\
Algeria & 1.13 \\
Egypt & 1.41 \\
\hline
\end{tabular}

to note that these projections should be interpreted with limitations. Because they are very sensitive to transmissibility of the virus, measured here by reproduction number over the projection period. If the reproduction number gets lower (than its current median level), the projected numbers of daily incidences will get lower and vice versa.

\section{Autoregressive Conditional Poisson Model}

Reproduction number discussed in section 3 measures mainly the short range dependence. To asses statistical significance of this short range dependence and also to study longer range dependence, a model that accounts for these and handles the type of data we have is discussed in this section.

Number of daily (new) infections is a count time series. Models employed to study dynamics of pandemics such as COVID-19 should take two important features of the variable (number of infections) into account: a) the variable is a discrete count variable $\rightarrow$ the usual Gaussian assumption doesn't hold, b) there is dependence among observations $\rightarrow$ uncorrelatedness of the observations, required by generalized linear models, will not be in place. A class of models known as autoregressive conditional poisson (ACP) models, discussed in recent literatures (e.g., [19] [20] [21] [22]), have attractive specifications that makes them capable to account for these issues and model count time series.

A log-linear version of this family of models has been proposed by [22] in order to accommodate modeling negative associations. This form of ACP has been used in some recent articles (e.g., [23] [24]) to study COVID-19 contagion. However, for count data such as new cases of COVID-19 pandemic, negative correlation could not make sense. Because expected number of cases at time $t$ has positive correlation with number of cases and mean of cases at time $t-1$. That means 
there is no need to worry about negative correlation in this situation. On the other hand, the log-linear version may have interpretational and inferential inconveniences for count data involving 0 's, which is common in daily new cases of COVID-19. Therefore, this study uses the original ACP model proposed by [19] and discussed in [21], given in Equation (1) below, which is an integer-valued analogue of the classical GARCH model.

Let $Y_{t}$ be a time dependent count time series (daily new cases of COVID-19 in this case) and let $\mathfrak{F}_{t-1}$ denotes the information available on the series up to time $t-1$. The autoregressive conditional Poisson model employed in this study is then given by

$$
\begin{gathered}
Y_{t} \mid \mathfrak{F}_{t-1} \sim \operatorname{Poisson}\left(\lambda_{t}\right), \text { for } \lambda_{t}=E\left(Y_{t} \mid \mathfrak{F}_{t-1}\right) \\
\lambda_{t}=\gamma+\alpha \lambda_{t-1}+\beta Y_{t-1}, t \geq 1
\end{gathered}
$$

where $\gamma$ is the intercept term, $\beta$ and $\alpha$ measure dependence of the expected number of new infections on day $t, \lambda_{t}$ on the past counts and past expected (mean) number of new infections respectively. Specifically, the $\beta$ component represents the short range serial dependence of $\lambda_{t}$ on the previous time observation and the $\alpha$ component represents the autoregresive feedback of mean of all past values of the daily new cases util day $t-1$ (can be referred to as long range dependence). All parameters $\gamma, \alpha$ and $\beta$ are assumed to be positive and satisfy $0<\alpha+\beta<1$ [22]. The analysis of this model is based on likelihood inference [21].

The notion of long range and short range dependence in this model is not the same as in classical time series models, which discuss dependence of time series observations on their own past values and other predictors. In the ACP model here, we deal with dependence of current conditional mean on its previous value and previous observation. In this case, if there is trend in observed time series of new cases, current mean can be affected more by the short range changes. If there is no trend in observed new cases, current mean is less affected by short range changes. This concept seems to have been misrepresented in some recent papers (e.g., [23]).

A brief assessment of daily new cases has shown that there is no serious overdisperssion problem in these count data. Therefore, a conditional Poisson distribution is used in this analysis as assumed in (1).

With the aim of testing significance of short range and long range dependences; and studying the variation of these dependences with time, the ACP model in (1) is fitted using different segments of data. First I fit the model using all data for each country. Estimates of the parameters and their $95 \%$ confidence intervals are given in Table 3. As can be seen here, the short range dependence is significant for all countries. This also strengthens reliability of the estimated reproduction numbers discussed in section 3 . Because reproduction numbers measure more of short range dependences.

Autoregressive feedback (long range serial dependence) at the end of this study period, June 15, is not significant for some of the countries (South Africa 
Table 3. Estimates of model parameters. Values in bracket are lower and upper limits of 95\% confidence interval. The estimates in bold are significant, at $95 \%$ level of significance.

\begin{tabular}{lccc}
\hline & \multicolumn{3}{c}{ Estimate } \\
\cline { 2 - 4 } Country & $\hat{\gamma}$ & $\hat{\alpha}$ & $\hat{\beta}$ \\
\hline South Africa & $\mathbf{8 2 . 8 4}(79.17,86.53)$ & $\sim 0(-0.01,0.01)$ & $\mathbf{0 . 9 3}(0.92,0.95)$ \\
DRC & $\sim 0(-0.01,0.01)$ & $\mathbf{0 . 8 1}(0.72,0.89)$ & $\mathbf{0 . 1 4}(0.06,0.23)$ \\
Gabon & $\sim 0(-0.01,0.01)$ & $\mathbf{0 . 8 4}(0.82,0.87)$ & $\mathbf{0 . 1 7}(0.14,0.17)$ \\
Nigeria & $\sim 0(-0.003,0.003)$ & $\mathbf{0 . 8 1}(0.79,0.84)$ & $\mathbf{0 . 1 9}(0.17,0.20)$ \\
Côte d'Ivoire & $\sim 0(-0.02,0.02)$ & $\mathbf{0 . 7 6}(0.72,0.81)$ & $\mathbf{0 . 2 4}(0.20,0.27)$ \\
Guinea & $\sim 0(-0.004,0.004)$ & $\mathbf{0 . 7 9}(0.76,0.83)$ & $\mathbf{0 . 2 1}(0.18,0.24)$ \\
Ethiopia & $\sim 0(-0.04,0.04)$ & $\mathbf{0 . 5 3}(0.44,0.62)$ & $\mathbf{0 . 4 6}(0.38,0.54)$ \\
Kenya & $0.58(0.20,0.96)$ & $\mathbf{0 . 4 2}(0.35,0.49)$ & $\mathbf{0 . 5 4}(0.48,0.61)$ \\
Sudan & $0.03(-0.01,0.07)$ & $\mathbf{0 . 7 5}(0.73,0.79)$ & $\mathbf{0 . 2 4}(0.21,0.27)$ \\
Djibouti & $\mathbf{0 . 5 8}(0.29,0.86)$ & $\mathbf{0 . 5 9}(0.56,0.62)$ & $\mathbf{0 . 3 7}(0.34,0.40)$ \\
Algeria & $\mathbf{3 7 . 6 4 ( 3 3 . 6 5 , 4 1 . 6 3 )}$ & $\mathbf{0 . 1 2}(0.06,0.19)$ & $\mathbf{0 . 5 4}(0.50,0.58)$ \\
Egypt & $\mathbf{1 8 . 3 2}(16.75,19.89)$ & $0.02(-0.004,0.05)$ & $\mathbf{0 . 9 3}(0.91,0.96)$ \\
\hline
\end{tabular}

and Egypt) indicating that there is no notable dependence on the mean number of cases of the preceding day. That in other words mean the expected number of cases on day $t$ is more explained by the number of new cases observed on day $t-$ 1 for those countries. That also likely explains the presence of continued upward trend in daily cases in these countries.

Interpretation of these estimates is direct forward. For instance in South Africa, where the short range dependence is consistently dominant: if the expected number of cases for today is 0 and 200 new cases observed on this day, then this leads to expected number of about 269 (95\% CI: 263 - 277) tomorrow. In countries where long range dependence has consistently been dominant, this expectation would be lower. For instance in Guinea: if the expected number of cases for today is 0 and 200 new cases observed, this leads to about 42 (95\% CI: 36 - 48) expected cases tomorrow. On the other hand if the expected number of cases is 200 and 0 new cases are reported today, this will lead to expected number of 83 (CI: 79 - 87) in South Africa and 158 (CI: 152 - 166) in Guinea the next day. This being the theoretical interpretation, in practice, however, assuming 0 conditional expectation in this case would be less realistic at this stage. Because such assumption could be valid only if probability of positive tests is zero. Therefore, more effort should be made towards minimizing observed number of daily new cases which in effect minimizes the expected (mean) numbers.

To study how the short range and long range dependences change with time, different models using increasing amounts of data are fitted for each country. The first fit is using daily new cases observed over the first 30 - 36 days, and then for each of the next fit including 5 days data at a time. For instance, the fist fit using observed new cases in the first 35 days, the second fit using number of new 
cases in first 40 days, the third fit using the first 45 days and so on. Plot of these estimates against sample size/time are given in Figure 4. Dominance of the two
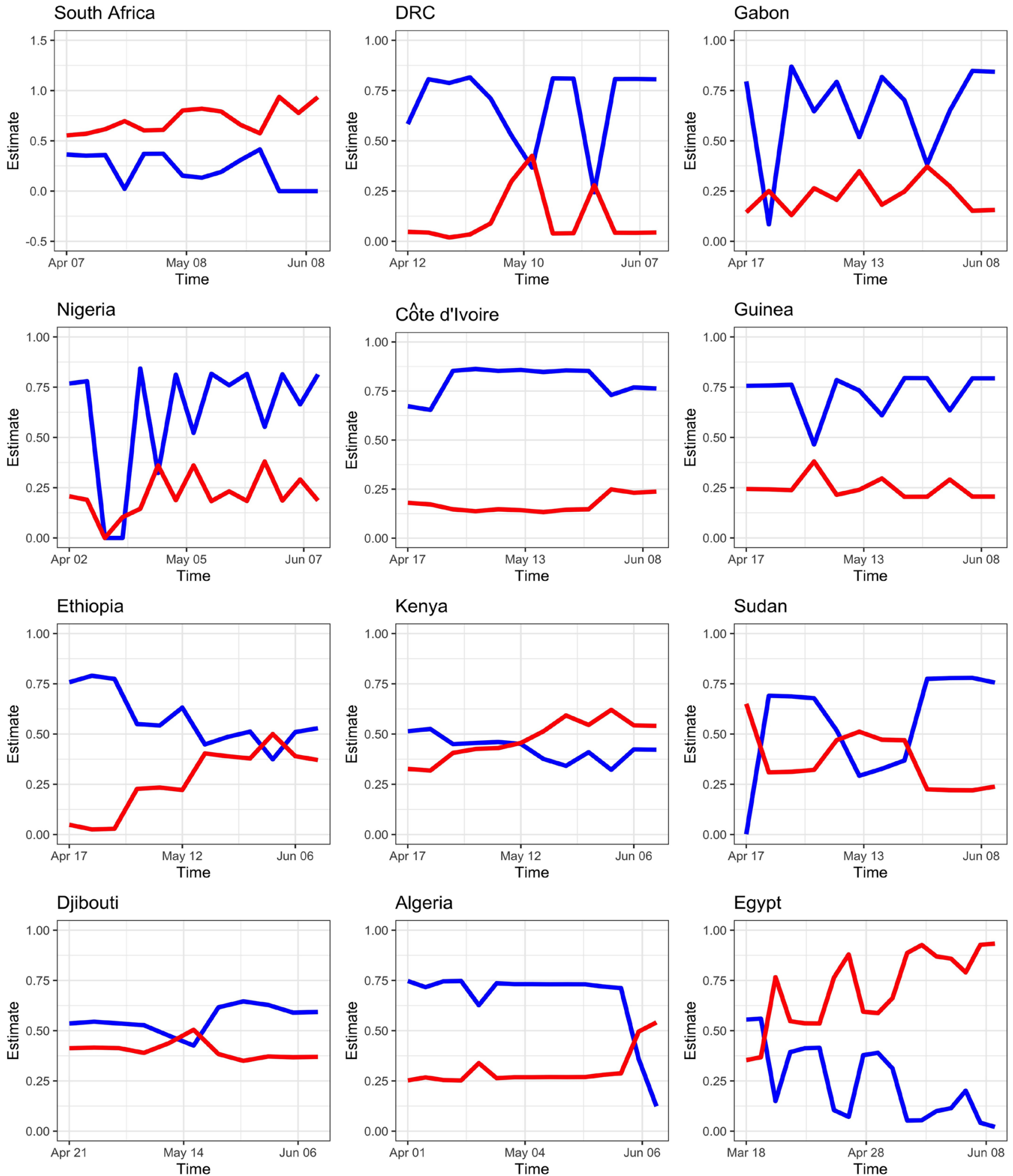

Figure 4. Estimates of the ACP model coefficients using sample sizes that increase with time. The red line represents estimates of coefficient that measures short range dependence, $\beta$, and the blue line represents estimates of coefficient that measures long range dependence, $\alpha$. 
dependences (measured by $\alpha$ and $\beta$ ) change when there is local (temporary) pause in trends during this segmented estimation time (after the first 30 - 36 days of the pandemic in each country). For instance, the continued dominance of long range dependence in DRC, Gabon, Côte d'Ivoire, Nigeria and Guinea (mostly West African countries) implies that there has not been a considerably continued trend after the first 30 - 36 days of first case in these countries. On the other hand, continued dominance of short range dependence, for instance in South Africa and Egypt, shows that there has been a persistent upward trend over the aforementioned period.

\section{Conflicts of Interest}

The author declares no conflicts of interest regarding the publication of this paper.

\section{References}

[1] World Health Organization (2020) WHO Director-General's Opening Remarks at the Media Briefing on COVID-19 11 March 2020.

https://www.who.int/dg/speeches/detail/who-director-general-s-opening-remarks-a t-the-media-briefing-on-covid-19---11-march-2020

[2] Kapata, N., Ihekweazu, C., Ntoumi, F., Raji, T., Chanda-Kapata, P., Mwaba, P., et al. (2020) Is Africa Prepared for Tackling the COVID-19 (SARS-CoV-2) Epidemic. Lessons from Past Outbreaks, Ongoing Pan-African Public Health Efforts, and Implications for the Future. International Journal of Infectious Diseases, 93, 233-236. https://doi.org/10.1016/j.ijid.2020.02.049

[3] World Health Organization (2020) Coronavirus Disease (COVID-2019) Situation Reports. https://www.who.int/emergencies/diseases/novel-coronavirus-2019/situation-reports

[4] Boëlle, P.Y., Ansart, S., Cori, A. and Valleron, A.J. (2011) Transmission Parameters of the A/H1N1 (2009) Influenza Virus Pandemic: A Review. Influenza and Other Respiratory Viruses, 5, 306-316. https://doi.org/10.1111/j.1750-2659.2011.00234.x

[5] Howard, S. and Donnelly, C. (2000) Estimation of a Time-Varying Force of Infection and Basic Reproduction Number with Application to an Outbreak of Classical Swine Fever. Journal of Epidemiology and Biostatistics, 5, 161-168.

[6] Riley, S., Fraser, C., Donnelly, C.A., Ghani, A.C., Abu-Raddad, L.J., Hedley, A.J., et al. (2003) Transmission Dynamics of the Etiological Agent of SARS in Hong Kong: Impact of Public Health Interventions. Science, 300, 1961-1966.

https://doi.org/10.1126/science.1086478

[7] Amundsen, E., Stigum, H., Røttingen, J.A. and Aalen, O. (2004) Definition and Estimation of an Actual Reproduction Number Describing Past Infectious Disease Transmission: Application to HIV Epidemics among Homosexual Men in Denmark, Norway and Sweden. Epidemiology \& Infection, 132, 1139-1149. https://doi.org/10.1017/S0950268804002997

[8] Cori, A., Ferguson, N.M., Fraser, C. and Cauchemez, S. (2013) A New Framework and Software to Estimate Time-Varying Reproduction Numbers during Epidemics. American Journal of Epidemiology, 178, 1505-1512. https://doi.org/10.1093/aje/kwt133

[9] He, X., Lau, E.H., Wu, P., Deng, X., Wang, J., Hao, X., et al. (2020) Temporal Dy- 
namics in Viral Shedding and Transmissibility of COVID-19. Nature Medicine, 26, 672-675. https://doi.org/10.1038/s41591-020-0869-5

[10] Wei, W.E., Li, Z., Chiew, C.J., Yong, S.E., Toh, M.P. and Lee, V.J. (2020) Presymptomatic Transmission of SARS-CoV-2 Singapore, January 23-March 16, 2020. Morbidity and Mortality Weekly Report, 69, 411. https://doi.org/10.15585/mmwr.mm6914e1

[11] Wu, J.T., Leung, K., Bushman, M., Kishore, N., Niehus, R., de Salazar, P.M., et al. (2020) Estimating Clinical Severity of COVID-19 from the Transmission Dynamics in Wuhan, China. Nature Medicine, 26, 506-510.

https://doi.org/10.1038/s41591-020-0822-7

[12] Li, Q., Guan, X., Wu, P., Wang, X., Zhou, L., Tong, Y., et al. (2020) Early Transmission Dynamics in Wuhan, China, of Novel Coronavirus-Infected Pneumonia. New England Journal of Medicine, 382, 1199-1207.

[13] Du, Z., Xu, X., Wu, Y., Wang, L., Cowling, B. and Meyers, L. (2020) The Serial Interval of COVID-19 from Publicly Reported Confirmed Cases. https://doi.org/10.1101/2020.02.19.20025452

[14] Zhao, S., Gao, D., Zhuang, Z., Chong, M., Cai, Y., Ran, J., et al. (2020) Estimating the Serial Interval of the Novel Coronavirus Disease (COVID-19): A Statistical Analysis Using the Public Data in Hong Kong from January 16 to February 15, 2020. https://doi.org/10.21203/rs.3.rs-18805/v2

[15] Bi, Q., Wu, Y., Mei, S., Ye, C., Zou, X., Zhang, Z., et al. (2020) Epidemiology and Transmission of COVID-19 in 391 Cases and 1286 of Their Close Contacts in Shenzhen, China: A Retrospective Cohort Study. The Lancet Infectious Diseases. https://doi.org/10.1016/S1473-3099(20)30287-5

[16] Ferguson, N.M., Cummings, D.A., Fraser, C., Cajka, J.C., Cooley, P.C. and Burke, D.S. (2006) Strategies for Mitigating an Influenza Pandemic. Nature, 442, 448-452. https://doi.org/10.1038/nature04795

[17] Roy, M., Son, A. and May, R.M. (1982) Directly Transmitted Infectious Diseases: Control by Vaccination. Science, 215, 1053-1060.

https://doi.org/10.1126/science.7063839

[18] Biggerstaff, M., Cauchemez, S., Reed, C., Gambhir, M. and Finelli, L. (2014) Estimates of the Reproduction Number for Seasonal, Pandemic, and Zoonotic Influenza: A Systematic Review of the Literature. BMC Infectious Diseases, 14, Article No. 480. https://doi.org/10.1186/1471-2334-14-480

[19] Heinen, A. (2003) Modelling Time Series Count Data: An Autoregressive Conditional Poisson Model. https://doi.org/10.2139/ssrn.1117187

[20] Ferland, R., Latour, A. and Oraichi, D. (2006) Integer-Valued GARCH Process. Journal of Time Series Analysis, 27, 923-942. https://doi.org/10.1111/j.1467-9892.2006.00496.x

[21] Fokianos, K., Rahbek, A. and Tjøstheim, D. (2009) Poisson Autoregression. Journal of the American Statistical Association, 104, 1430-1439. https://doi.org/10.1198/jasa.2009.tm08270

[22] Fokianos, K. and Tjøstheim, D. (2011) Log-Linear Poisson Autoregression. Journal of Multivariate Analysis, 102, 563-578. https://doi.org/10.1016/j.jmva.2010.11.002

[23] Agosto, A. and Giudici, P. (2020) A Poisson Autoregressive Model to Understand COVID-19 Contagion Dynamics. https://doi.org/10.2139/ssrn.3551626

[24] Kharroubi, S. (2020) Modeling and Predicting the Spread of COVID-19 in Lebanon: A Bayesian Perspective. https://doi.org/10.1101/2020.04.29.20082263 